Hydraulik und Pneumatik 
Holger Watter

\section{Hydraulik und Pneumatik}

Grundlagen und Übungen Anwendungen und Simulation

4., überarbeitete und erweiterte Auflage

Springer Vieweg 
Holger Watter

FH Flensburg

Flensburg, Deutschland

ISBN 978-3-658-07859-1

ISBN 978-3-658-07860-7 (eBook)

DOI 10.1007/978-3-658-07860-7

Die Deutsche Nationalbibliothek verzeichnet diese Publikation in der Deutschen Nationalbibliografie; detaillierte bibliografische Daten sind im Internet über http://dnb.d-nb.de abrufbar.

Springer Vieweg

(C) Springer Fachmedien Wiesbaden 2015

Das Werk einschließlich aller seiner Teile ist urheberrechtlich geschützt. Jede Verwertung, die nicht ausdrücklich vom Urheberrechtsgesetz zugelassen ist, bedarf der vorherigen Zustimmung des Verlags. Das gilt insbesondere für Vervielfältigungen, Bearbeitungen, Übersetzungen, Mikroverfilmungen und die Einspeicherung und Verarbeitung in elektronischen Systemen.

Die Wiedergabe von Gebrauchsnamen, Handelsnamen, Warenbezeichnungen usw. in diesem Werk berechtigt auch ohne besondere Kennzeichnung nicht zu der Annahme, dass solche Namen im Sinne der Warenzeichen- und Markenschutz-Gesetzgebung als frei zu betrachten wären und daher von jedermann benutzt werden dürften.

Der Verlag, die Autoren und die Herausgeber gehen davon aus, dass die Angaben und Informationen in diesem Werk zum Zeitpunkt der Veröffentlichung vollständig und korrekt sind. Weder der Verlag noch die Autoren oder die Herausgeber übernehmen, ausdrücklich oder implizit, Gewähr für den Inhalt des Werkes, etwaige Fehler oder Äußerungen.

Lektorat: Thomas Zipsner

Gedruckt auf säurefreiem und chlorfrei gebleichtem Papier.

Springer Fachmedien Wiesbaden GmbH ist Teil der Fachverlagsgruppe Springer Science+Business Media (www.springer.com) 


\section{Danksagung}

Eine Vorlesung mit anschaulichen Beispielen vorzubereiten, erfordert mehr Arbeit, als Studierende oder Laien erahnen: Viele Stunden Recherche, Aufarbeitung von Gefundenem, Verwerfen von Grafiken und Beispielen, die sich in der Lehre nicht bewährt haben. All dies geschieht im Verborgenen und wird vom „Kunden“ stillschweigend mit hoher Aktualität erwartet.

Aus einer erprobten Vorlesung ein Buch zu machen, müsste dann doch wohl relativ einfach sein! Weit gefehlt! Die Stichworte aus der Vorlesung müssen ausformuliert sein; Dinge, die aus der Erfahrung ,eben mal aus dem Handgelenk geschüttelt“ und vorgetragen werden, müssen nun didaktisch eingebunden werden; handschriftliche Folien und Tafelskizzen müssen fürs Layout elektronisch aufbereitet werden ... wieder mehr Arbeit als man denkt.

Ich möchte mich daher bei Menschen bedanken, die mich bei der Arbeit und im Rahmen von Projekten unterstützt haben:

- Herr Dipl.-Ing. Stefan Claußen hat bei zurückliegenden Buchprojekten bereits hilfreiche Arbeit geliefert. Insbesondere die Teile über die Schmierstoffeigenschaften basieren auf dieser Zusammenarbeit!

- Frau Dr.-Ing. Sylvia Ullmer hat mich bei der Durchführung des EU-Projektes LLINCWA (loss/lost lubrication in coastal and inland water activities) tatkräftig unterstützt. Der Teì zu den biologisch-äb-̄aubaren Schmierstoffen entstand aus dieser Zusammenarbeit.

- Herr Dipl.-Ing. Siegfried Prust stand als Mitarbeiter des Labors für Hydraulik und Pneumatik (H\&P) an der Hochschule für Angewandte Wissenschaft (HAW) Hamburg immer wieder beratend zur Seite. Offene Fragen und Lösungsansätze konnten durch Laborversuche schnell und unkompliziert evaluiert werden. Ich danke insbesondere für die Anfertigung der Fotos.

- Bei Herrn Dipl.-Ing. Thomas Zipsner bedanke ich mich für die Initiative zu diesem Buch, das Vertrauen, die Beratung und die tatkräftige Unterstützung bei der Umsetzung.

- Bei der Hochschule für Angewandte Wissenschaften (HAW) Hamburg bedanke ich mich für die Bereitstellung der Laborkapazitäten sowie der Ressourcen und Kompetenzen. 
- Bei meiner Familie, insbesondere bei meiner Frau Petra Watter, bedanke ich mich für ihr Verständnis und die Unterstützung. Zahlreiche Stunden am PC (oft bis in die späte Nacht und am Wochenende) wurden ohne Murren akzeptiert.

- „Last but not least“ bei den Studierenden für Verbesserungs- und Korrekturvorschläge zur 2. bis 4. Auflage!

Tarp, Februar 2015

Holger Watter 


\section{Vorwort}

Die Fluidtechnik ist eine interdisziplinäre Paradedisziplin des Maschinenbaus. Aufbauend auf Grundlagenkenntnisse aus den Bereichen

- der technischen Mechanik (Belastungen, Dimensionierung, Spezifikation),

- der Betriebsstofflehre (Eigenschaften und Charakteristika der Fluide),

- der Strömungslehre und Thermodynamik (kompressible, inkompressible Medien, Zustandsänderungen, Strömungsverluste) sowie

- der Mess-, Steuerungs- und Regelungstechnik (Automatisierung, Peripherie)

werden vertiefende Kenntnisse aus den Bereichen

- der Systemtechnik (Schnittstellenproblematik, Interaktionen der Systeme, Simulationstechnik) und

- der Anlagen- und Antriebstechnik benötigt (Verdrängerpumpen, -maschinen und -kompressoren, Hydro- und Druckluftmotoren, Wandler, hydrodynamische -Kupplungen).

Die Gliederung dieses Buches orientiert sich an diesen Anforderungen. Es wendet sich an angehende Ingenieure und Ingenieurinnen in der Bachelor- und Masterausbildung sowie Praktiker im Betrieb. Nach der Darstellung des Grundlagenwissens zu den Betriebsstoffen (Kap. 2) und zur Fluidmechanik (Kap. 3) werden die wichtigsten Systemkomponenten und deren Wirkmechanismen vorgestellt (Kap. 4). Zahlreiche Beispiele verdeutlichen exemplarisch diese Zusammenhänge. Dabei steht weniger die Konstruktion ausgewählter Bauteile im Vordergrund, sondern vielmehr das Verständnis um die komplexen Wirkzusammenhänge und die Systemdynamik: Messen - Steuern - Regeln Antreiben: Keine „Schräubchenkunde“ - Beschränkung auf die ingenieurgerechte Beschreibung der Wirkmechanismen. Es wird das verallgemeinerte Betriebsverhalten und die Betriebscharakteristik der mechatronischen Komponenten vorgestellt, soweit diese für die Anlagenkonfiguration, den Betrieb und die Störungssuche hilfreich sein können (Kap. 4). Konstruktive Details können den umfangreichen Web-Seiten der Komponentenhersteller entnommen werden; eine Auswahl dazu wird auf der Verlagsseite als OnlineService (vgl. Anhang A6) bereitgehalten. 
Obwohl der Schwerpunkt des Buches wegen der besonderen Bedeutung der Mobilhydraulik auf dem Gebiet der Ölhydraulik liegt, wird immer wieder versucht, Parallelen und Differenzen zum Systemverhalten der Pneumatik herauszuarbeiten.

Einen besonderen Schwerpunkt bildet dabei die moderne Simulationstechnik (Kap. 5), hier soll eine erkannte Lücke in der deutschsprachigen Fachliteratur geschlossen werden. Die dazu notwendigen mathematischen Grundkenntnisse werden in den vorgenannten Abschnitten konsequent gelegt sowie dazu wichtige Randgebiete in den Anhängen A1 bis A4 behandelt. Anhand von anschaulichen Beispielen aus der Fluidtechnik wird die Herangehensweise zur Lösung von interdisziplinären dynamischen Problemstellungen vorgestellt. Durch Kenntnisse in der Simulationstechnik kann der Leser die Ergebnisse von kommerziellen Softwaretools kritisch hinterfragen - eine fachliche Qualifikation, die zunehmend wichtiger für die berufliche Praxis wird.

Die vorliegende 4. Auflage wurde als E-Book grundständig überarbeitet und ergänzt um ein Abkürzungsverzeichnis, didaktisch-methodische Verbesserungen, angepasstes Layout (z. B. durch farbige Abbildungen) sowie vertiefenden Inhalte zur Auslegung von Hydrospeichern, weitere Beispiele zur Simulations- und Visualisierungstechnik (nun auch in SCILAB). 


\section{Formelzeichen und Abkürzungen}

\section{Formelzeichen}

\begin{tabular}{|c|c|}
\hline$a$ & Schallgeschwindigkeit $[\mathrm{m} / \mathrm{s}]$ \\
\hline$A$ & Fläche $\left[\mathrm{m}^{2}\right]$ \\
\hline$B$ & magn. Induktion $\left[\mathrm{V} s / \mathrm{m}^{2}=\mathrm{T}\right]$ \\
\hline$b$ & Dämpfungskonstante $[\mathrm{N} /(\mathrm{m} / \mathrm{s})]$ \\
\hline$c$ & Strömungsgeschwindigkeit [m/s] \\
\hline$c_{p}$ & spez. Wärmekapazität [J/kg K] \\
\hline$C$ & Kapazität [m³/bar] \\
\hline$D$ & Dämpfungsgrad \\
\hline$D$ & Geschwindigkeitsgefälle $\left[\mathrm{s}^{-1}\right]$ \\
\hline e & EULER-Zahl: $e=2,71828$ \\
\hline$f$ & Erregerfrequenz $[\mathrm{Hz}=1 / \mathrm{s}]$ \\
\hline$f_{0}$ & Eigenfrequenz $[\mathrm{Hz}=1 / \mathrm{s}]$ \\
\hline$F$ & $\operatorname{Kraft}[\mathrm{N}]$ \\
\hline$g$ & Erdbeschleunigung, Gravitationskonst. $9,81 \mathrm{~m} / \mathrm{s}^{2}$ \\
\hline$G(s)$ & Übertragungsfunktion \\
\hline$G$ & $\begin{array}{l}\text { (laminarer/turbulenter) Leitwert [(Ltr/min }) / \text { bar }] \\
{\left[(\mathrm{Ltr} / \mathrm{min})^{2} / \mathrm{bar}\right]}\end{array}$ \\
\hline$H$ & Feldstärke $[\mathrm{A} / \mathrm{cm}]$ \\
\hline $\operatorname{Im}$ & Imaginärteil einer komplexen Zahl \\
\hline$J$ & Massenträgheitsmoment $\left[\mathrm{kg} \mathrm{m}^{2}\right]$ \\
\hline$K$ & Kompressionsmodul [bar] \\
\hline$L$ & Induktivität [bar/(Ltr/min)/s] \\
\hline$m$ & Masse $[\mathrm{kg}]$ \\
\hline$\dot{m}$ & Massenstrom [kg/s] \\
\hline$M$ & Drehmoment $[\mathrm{Nm}]$ \\
\hline$M$ & molare Masse [kg/kmol] \\
\hline$p$ & Druck [bar] \\
\hline
\end{tabular}




\begin{tabular}{|c|c|}
\hline$P$ & Leistung $[\mathrm{W}, \mathrm{kW}]$ \\
\hline$Q=\dot{V}$ & Volumenstrom $\left[\mathrm{m}^{3} / \mathrm{s}, \mathrm{Ltr} / \mathrm{min}\right]$ \\
\hline $\operatorname{Re}$ & REYNOLDs-Zahl \\
\hline $\operatorname{Re}$ & Realteil einer komplexen Zahl \\
\hline$\Re$ & allg. Gaskonstante $8,314 \mathrm{~kJ} / \mathrm{kmol} \mathrm{K}$ \\
\hline $\mathrm{R}$ & spez. Gaskonstante $[\mathrm{J} / \mathrm{kg} \mathrm{K}]$ \\
\hline$R_{\text {lam }}$ & laminarer Widerstand [bar/(Ltr/min)] \\
\hline$R_{\text {turb }}$ & turbulenter Widerstand $\left[\mathrm{bar} /(\mathrm{Ltr} / \mathrm{min})^{2}\right]$ \\
\hline$s$ & Wandstärke [mm] \\
\hline$s$ & Sollwert [\%] \\
\hline$s=\frac{\mathrm{d}}{\mathrm{d} t}$ & LAPLACE-Operator \\
\hline $\mathrm{W}$ & Arbeit $[\mathrm{Nm}=\mathrm{J}=\mathrm{Ws}]$ \\
\hline VI & Viskositätsindex \\
\hline$V$ & Verstärkungsfunktion, Amplitudengang \\
\hline$V$ & Volumen $\left[\mathrm{m}^{3}\right]$ \\
\hline$\dot{V}=\frac{\mathrm{d} V}{\mathrm{~d} t}$ & Volumenänderung $\left[\mathrm{m}^{3} / \mathrm{s}\right]$ \\
\hline$V_{H}$ & Schluckvolumen $\left[\mathrm{cm}^{3}\right]$ \\
\hline$x$ & Wegposition [m] \\
\hline$\dot{x}$ & Geschwindigkeit $[\mathrm{m} / \mathrm{s}]$ \\
\hline$\ddot{x}$ & Beschleunigung $\left[\mathrm{m} / \mathrm{s}^{2}\right]$ \\
\hline$z$ & Zylinderzahl, Zähnezahl \\
\hline$\alpha$ & Winkel [rad] \\
\hline$\alpha$ & Durchflusszahl (Strömungseinschnürung) \\
\hline$\alpha_{L}$ & BUNSEN-Koeff. \\
\hline$\beta=\frac{1}{K}$ & Kompressibilität/Pressziffer [1/bar] \\
\hline$\beta_{10}$ & Filterfeinheit \\
\hline$\delta$ & Abklingkonstante $[1 / \mathrm{s}]$ \\
\hline$\delta$ & Ungleichförmigkeitsgrad \\
\hline$\varepsilon$ & Dehnung \\
\hline$\eta$ & Wirkungsgrad \\
\hline$\eta$ & dynamische Viskosität [Pa s] \\
\hline$\varphi$ & Drehwinkel [rad] \\
\hline$\dot{\varphi}=\omega$ & Winkelgeschwindigkeit [rad/s] \\
\hline$\ddot{\varphi}=\dot{\omega}$ & Winkelbeschleunigung $\left[\mathrm{rad} / \mathrm{s}^{2}\right]$ \\
\hline$\lambda$ & Liefergrad \\
\hline$\mu$ & magn. Permeabilität $[\Omega \mathrm{s} / \mathrm{m}]$ \\
\hline$\mu$ & Haftungs-/Reibungsbeiwert \\
\hline$\kappa$ & Isentropenexponent \\
\hline$v$ & kinematische Viskosität $\left[\mathrm{mm}^{2} / \mathrm{s}\right]$ \\
\hline$v$ & Querkontraktionszahl, POISSON-Zahl \\
\hline$\rho$ & Dichte $\left[\mathrm{kg} / \mathrm{m}^{3}\right]$ \\
\hline$\sigma$ & Normalspannungen $\left[\mathrm{N} / \mathrm{mm}^{2}\right]$ \\
\hline
\end{tabular}




$\begin{array}{ll}\tau=t & \text { Zeit }[\mathrm{s}] \\ \tau & \text { Schubspannung }\left[\mathrm{N} / \mathrm{mm}^{2}\right] \\ \omega & \text { Winkelgeschwindigkeit }[\mathrm{rad} / \mathrm{s}] \\ \omega_{0} & \text { Eigenkreisfrequenz }[\mathrm{rad} / \mathrm{s}] \\ \Omega & \text { Erregerkreisfrequenz }[\mathrm{rad} / \mathrm{s}] \\ \zeta & \text { Widerstandsbeiwert } \\ \Psi & \text { Durchflussfunktion (Druckverhältnis vor/hinter Düse, Blende o. Ä.) }\end{array}$

\section{Abkürzungen}

$\begin{array}{ll}\text { API } & \text { American Petroleum Institute (u. a. Klassifizierung von Ölen) } \\ \text { ATF } & \text { Automatic Transmission Fluid } \\ \text { BN } & \text { Base Number = Basenzahl } \\ \text { CAN } & \text { Controller Area Network (Bussystem) } \\ \text { CEC } & \text { Coordinating European Council (Normenausschuss u. a. für Schmierstoffe) } \\ \text { COC } & \text { CLEVELAND open cup (Flammpunkt im offenen Tiegel) } \\ \text { dB(A) } & \text { dezi-Bell-A = A-bewerteter Schalldruckpegel (log.-Verhältnis) } \\ \text { DIN } & \text { Deutsches Institut für Normung } \\ \text { EP } & \text { Extreme-Pressure-Zusätze verschleißmindernd u. a. für hohe Flächenpres- } \\ & \text { sungen } \\ \text { H... } & \text { Normbezeichnung Hydrauliköl } \\ \text { HD } & \text { High Duty (Schmieröl u. a. mit Extreme-Pressure-Zusätze) } \\ \text { HL, HLP } & \text { Normbezeichnung für legierte Hydrauliköle (vgl. Kap. 2) } \\ \text { HF... } & \text { Normbezeichnung für schwer entflammbare Hydrauliköle } \\ \text { HE... } & \text { Normbezeichnung für biolog. schnell abbaubare Hydrauliköle } \\ \text { HEES } & \text { Hydraulic Environmental Ester Synthetic } \\ \text { HEPG } & \text { Hydraulic Environmental Polyglycol } \\ \text { HETG } & \text { Hydraulic Environmental Triglyceride } \\ \text { ISO } & \text { International Standardisation Organisation } \\ \text { LS } & \text { Load Sensing (= feinfühlige Steuerung mit geringen Verlusten) } \\ \text { NZ } & \text { Neutralisationszahl } \\ \text { OECD } & \text { Organisation für wirtschaftliche Zusammenarbeit und Entwicklung } \\ \text { PAO } & \text { Polyalphaolefine } \\ \text { PEG } & \text { Polyethylengycol } \\ \text { PIB } & \text { Pyly-Iso-Buten } \\ \text { PMCC } & \text { PENSKY-MARTENS close cup (Flammpunkt im geschlossenen Tiegel) } \\ \text { PPG } & \text { Polypropylenglykol } \\ \text { SAE } & \text { Society of Automotive Engineers (u. a. Schmierstoffklassifizierung) } \\ \text { SPS } & \text { Speicherprogrammierbare Steuerung } \\ \text { TAN } & \text { Total Acid Number (Säurezahl) } \\ \text { TBN } & \text { Total Base Number (auch BN = Base Number = Basenzahl) } \\ & \end{array}$


VDMA Verband Deutscher Maschinen und Anlagenbauer

VG Viscosity Grade (Viskositätsklasse)

VI Viskositäts-Index

VwVsS Verwaltungsvorschrift wassergefährdender Stoffe

WGK Wassergefährdungsklasse 


\section{Inhaltsverzeichnis}

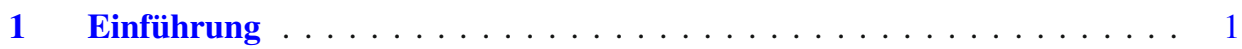

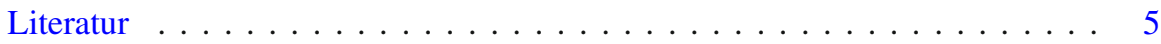

2 Fluide und Fluideigenschaften $\ldots \ldots \ldots \ldots \ldots \ldots \ldots$

2.1 Physikalisch-chemische Eigenschaften der Druckflüssigkeiten $\ldots \ldots 7$

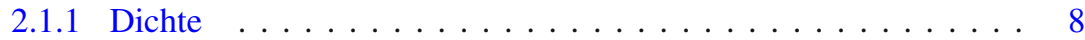

2.1 .2 Viskosität . . . . . . . . . . . . . . . . . 10

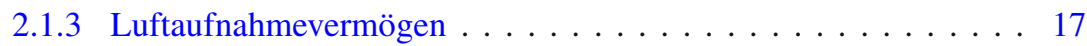

2.1.4 Wassergehalt ...................... 18

2.1.5 Flammpunkt/Brennpunkt . . . . . . . . . . . . . . . . 19

2.1.6 Aschegehalt, Verkokungsrückstand . . . . . . . . . . . . . . 19

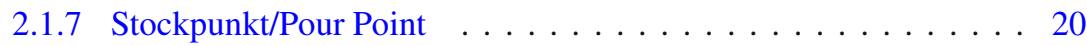

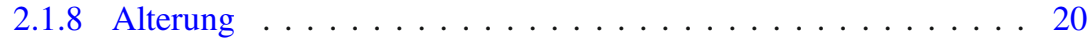

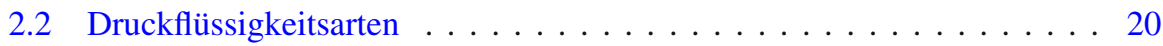

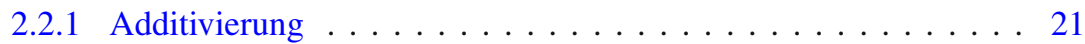

2.2.2 Synthetische Schmierstoffe . . . . . . . . . . . . . 25

2.2 .3 Klassifikation/Normung . . . . . . . . . . . . . . . 28

2.3 Biologisch abbaubare Hydraulikflüssigkeiten . . . . . . . . . 35

2.3.1 Problemfelder: Verlustschmierungen und technische Havarien . . 35

2.3.2 Betriebstechnisches Umfeld: Juristische Bewertung . . . . . . 36

2.3.3 Was sind umweltverträgliche Schmierstoffe? . . . . . . . . . 36

2.3.4 Erfahrungen/Stand der Technik . . . . . . . . . . . . . . 41

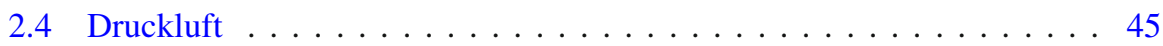

2.4.1 Stoffwerte von Luft $\ldots \ldots \ldots \ldots \ldots \ldots \ldots \ldots$

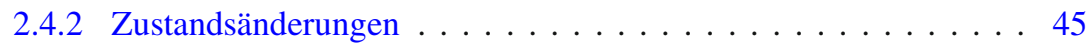

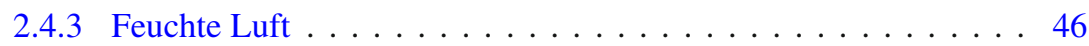

2.5 Übungsbeispiele . . . . . . . . . . . . . . . . . . . . . . . . . 49

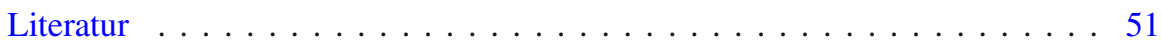

3 Grundlagen der Fluidmechanik $\ldots \ldots \ldots \ldots \ldots \ldots$

3.1 Kontinuitätsgleichung $\ldots \ldots \ldots \ldots \ldots \ldots \ldots \ldots \ldots$ 
3.2 Leistung/Energie/Satz von Bernoulli $\ldots \ldots \ldots \ldots \ldots \ldots$

3.3 Druckverluste $($ Strömungsverluste $R$ ) . . . . . . . . . . . . . . . 59

3.3.1 Strömungsverluste in geraden Rohrleitungen . . . . . . . . . . . . 60

3.3.2 Strömungsverluste an Einbauten und Ventilen . . . . . . . . . . . . 62

3.3.3 Reihen- und Parallelschaltung von Ventilen und Einbauten . . . . 64

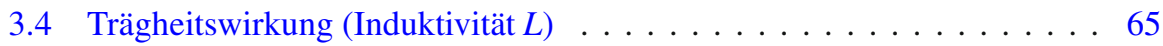

3.4.1 Beschleunigung des Fluids . . . . . . . . . . . . . . . . . 66

3.4 .2 Induktivität $L$ einer Rohrleitung . . . . . . . . . . . . . . . 66

3.4.3 Berücksichtigung der Trägheit von mitbewegten Bauteilen . . . . 67

3.4.4 Berücksichtigung der Trägheit bei rotatorischen Hydraulik-

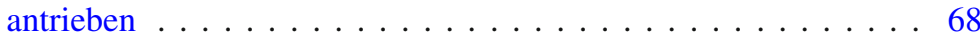

3.5 Kompressibilität $($ Kapazität $C) \ldots \ldots \ldots \ldots$. . . . . . . . . . . . . . . . . . . . . . . . 69

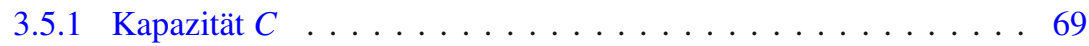

3.5.2 Hydraulische Kapazität einer Rohrleitung . . . . . . . . . . . 70

3.6 Kraftwirkungen strömender Flüssigkeiten/Impulssatz . . . . . . . . . . . 71

3.7 Leckverluste/Volumenstrom durch Drosselung $Q \ldots \ldots$. . . . . . . . 72

3.7.1 Leckströmungen infolge von Druckdifferenzen im parallelen Spalt 73

3.7.2 Leckstrom im Ringspalt . . . . . . . . . . . . . . . . . 75

3.7.3 Leckströmungen in röhrenförmigen Strömungskanälen . . . . . 75

3.7.4 Ausfluss an Drosselstellen (Pneumatik) . . . . . . . . . . . . . . 75

3.8 Schallgeschwindigkeit (Druckwellengeschwindigkeit) . . . . . . . . 78

3.9 Simulationsbeispiel . . . . . . . . . . . . . . . . . . . . . 79

3.10 Übungen und Beispiele $\ldots \ldots \ldots$. . . . . . . . . . . . . . . . . . . . . . . . . . . . . 80

Literatur . . . . . . . . . . . . . . . . . . . . . . . 84

4 Komponenten und Bauteile $\ldots \ldots \ldots \ldots \ldots$

4.1 Grundprinzip, Leistungsübertragung und Energiewandlung . . . . . . . 85

4.2 Statische Anlagenkennlinie . . . . . . . . . . . . . . . . . . . 90

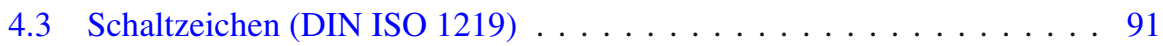

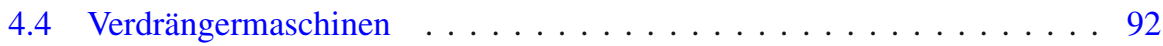

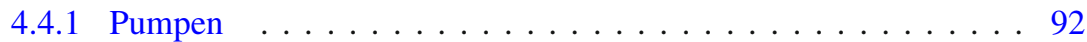

4.4 .2 Hubkolbenverdichter . . . . . . . . . . . . . 105

4.5 Ventile . . . . . . . . . . . . . . . . . . . . . 110

4.5 .1 Hydraulikventile . . . . . . . . . . . . . . . . . . . . . . . . . . . . . . . . . . . . . . . . . . .

4.5 .2 Pneumatikventile . . . . . . . . . . . . . . . . 138

4.6 Linear- und Schwenkmotoren (Aktoren) . . . . . . . . . . . . . . . . 144

4.6 .1 Hydromotoren . . . . . . . . . . . . . . . . . . . . 144

4.6.2 Pneumatikzylinder . . . . . . . . . . . . . . 155

4.7 Hydrostatische Antriebe/hydrodyn. Getriebe und Wandler . . . . . . . . 156

4.7.1 Hydrostatische Antriebe . . . . . . . . . . . . . . . 157

4.7 .2 Hydrodynamische Antriebe . . . . . . . . . . . . . . . . . . . . . . . . . . . . . . . . . . . . . . .

4.8 Zubehör . . . . . . . . . . . . . . . . . . . . . . . . . . . 164 
4.8 .1 Rohre, Schläuche . . . . . . . . . . . . . . . . . . . . . . 164

4.8 .2 Speicher . . . . . . . . . . . . . . . 166

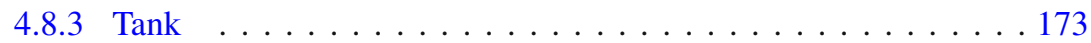

4.8 .4 Filter . . . . . . . . . . . . . . . . . . 175

4.8 .5 Kühler . . . . . . . . . . . . . . . . 176

4.8 .6 Schalldämpfer (Pneumatik) . . . . . . . . . . . . . 177

4.9 Simulationsbeispiel . . . . . . . . . . . . . . . . . . . 181

4.10 Visualisierung von Widerstand und Leitwert eines Regelventils . . . . . 183

4.11 Übungen und Beispiele . . . . . . . . . . . . . . . . . . . . 185

4.11 .1 Pumpen und Verdichter . . . . . . . . . . . . . . . . . . . . . . . . . . . . . . . . . . . .

4.11 .2 Hydraulische Maschinen . . . . . . . . . . . . . . . . . 186

4.11.3 Zusammenwirken von Pumpe und Anlage (Anlagenkennlinie) . . 186

4.11.4 Zylinder und Schwenkmotoren . . . . . . . . . . . . . . . 187

4.11 .5 Ventile und Einbauten . . . . . . . . . . . . . . . . . . 188

4.11.6 Wärmeverluste/Kühlerdimensionierung . . . . . . . . . . . . . . 190

4.11 .7 Speicher . . . . . . . . . . . . . . . . . . . . . 191

4.11 .8 Dynamische Vorgänge . . . . . . . . . . . . . . . . . . . . . . . . . . . . . . . . . . . . . . . . .

Literatur . . . . . . . . . . . . . . . . . . . . 192

5 Steuern, Regeln, Simulieren _. . . . . . . . . . . . . . . . . 195

5.1 Steuerungen . . . . . . . . . . . . . . . . . . 195

5.1.1 Konventioneller Aufbau logischer Schaltungen . . . . . . . . . . 197

5.1 .2 Speicherprogrammierbare Steuerungen (SPS) . . . . . . . . . . 198

5.1 .3 Bussysteme . . . . . . . . . . . . . . . . . 200

5.2 Regelungen . . . . . . . . . . . . . . . . . . . 206

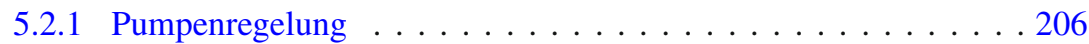

5.2 .2 Sekundärregelung . . . . . . . . . . . . . . 210

5.2.3 Positionierung einer hydraulischen Achse . . . . . . . . . . . . 214

5.3 Modellbildung und Simulation . . . . . . . . . . . . . . . 215

5.4 Übungen und Beispiele . . . . . . . . . . . . . . . . . . . . . . . . . . . . . . . . . . . . . . . . . . . . . . . . 25

Literatur . . . . . . . . . . . . . . . . . . . 226

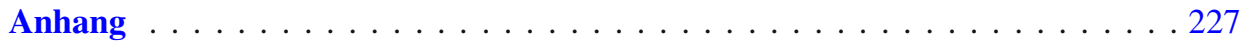

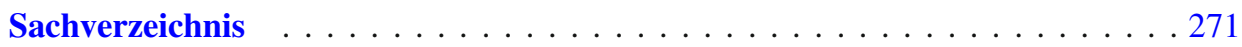

